Research Paper

\title{
Up-regulation and Pre-activation of TRAF3 and TRAF5 in Inflammatory Bowel Disease
}

\author{
Jun Shen, Yu-qi Qiao, Zhi-hua Ran ${ }^{\bowtie}$, Tian-rong Wang \\ Division of Gastroenterology and Hepatology, Shanghai Jiao-Tong University School of Medicine Renji Hospital, Shanghai Institute of Di- \\ gestive Disease; Key Laboratory of Gastroenterology \& Hepatology, Ministry of Health (Shanghai Jiao-Tong University). 145 Middle Shan- \\ dong Rd, Shanghai 200001, China.
}

\begin{abstract}
$\triangle$ Corresponding author: Zhi-hua Ran, E-mail: ranzhihua62@sina.cn. Telephone: +86 2163260930 Fax: +86 2163266027.
(c) Ivyspring International Publisher. This is an open-access article distributed under the terms of the Creative Commons License (http://creativecommons.org/ licenses/by-nc-nd/3.0/). Reproduction is permitted for personal, noncommercial use, provided that the article is in whole, unmodified, and properly cited.
\end{abstract}

Received: 2012.10.27; Accepted: 2012.12.24; Published: 2013.01.03

\begin{abstract}
Objective: TRAF3 and TRAF5 share a common ancestral gene, and interact as essential components of signaling pathways in immunity. TRAF3 and TRAF5 are overexpressed in the colon of rat/mouse models with colitis. However, the expressions of TRAF3 and TRAF5 in patients with inflammatory bowel disease have not been elucidated. The aim of the present study is to explore the potential roles of TRAF3 and TRAF5 in patients with inflammatory bowel disease.

Methods: Plasma levels of TRAF3 and TRAF5 proteins were detected by Enzyme-linked Immunosorbent Assay (ELISA). Colonic expression of TRAF3 and TRAF5 proteins was detected by western blot analysis. Quantitative Real-time PCR (qRT-PCR) was applied for gene expression. Inflamed intestinal mucosa and non-inflamed intestinal mucosa in patients with inflammatory bowel disease and normal mucosa was analyzed from healthy controls.

Results: The plasma levels of TRAF3 and TRAF5 were significantly higher both in patients with Crohn's disease and ulcerative colitis than in healthy controls. Only soluble TRAF5 showed a weak correlation with endoscopic disease activity index (Baron score) in patients with ulcerative colitis (spearman's $r=0.358, P=0.022$ ). Gene expressions of TRAF3 and TRAF5 in peripheral blood mononuclear cells were significantly higher both in patients with Crohn's disease and ulcerative colitis than in healthy controls (all $P<0.000 \mathrm{I}$ ). Gene and protein expressions of TRAF3 and TRAF5 were significantly higher in inflamed colonic mucosa of patients with Crohn's disease and ulcerative colitis than in non-inflamed colonic mucosa and normal mucosa of healthy controls (all $P<0.000 \mathrm{I}$ ). Furthermore, gene and protein expressions of TRAF3 and TRAF5 were also significantly higher in non-inflamed colonic mucosa of patients with Crohn's disease and ulcerative colitis than in normal mucosa of healthy controls.

Conclusions: TRAF3 and TRAF5 are overexpressed in inflammatory bowel disease. Although the endoscopic appearance can be normal, TRAF3 and TRAF5 pre-activation can be detected in non-inflamed colonic segments.
\end{abstract}

Key words: TRAF3; TRAF5; Crohn's disease; Ulcerative colitis.

\section{Introduction}

Tumor necrosis factor receptor (TNFR)associated factors (TRAFs) are emerging as essential components of signaling pathways in adaptive and innate immunity [1]. A wide range of signal pathways are mediated by TRAFs involving activation of nuclear factor $\mathrm{\kappa B}(\mathrm{NF}-\mathrm{\kappa B})$, mitogen-activated protein kinase (MAPK) or Toll-like receptors (TLRs) cascades $[2,3]$. In recent years, there has been great interest in 
the roles of TRAF3 and TRAF5 play in cells of the immune system [4]. TRAF3 and TRAF5 share a common ancestral gene and are therefore homologous [5]. It is shown that TRAF5 plays an important role in $B$ cell function, which requires TRAF3 to associate with endogenous LMP1 in B cells [6]. Although TRAF3 and TRAF5 are all involved in NF-kB activation, it seems that TRAF5 participates in the regulation of canonical and non-canonical NF-kB pathways [7], whereas TRAF3 is mainly involved in the control of non-canonical NF-kB signaling and has not been reported to contribute to the canonical pathway. Thus, additional roles for TRAF3 and TRAF5 associations are to be forthcoming.

TRAF3 and TRAF5 signals elicit immune responses and have important implications for disorders such as inflammatory bowel disease. In the colon and small intestine of experimental rats induced by trinitrobenzene sulfonic acid (TNBS), both TRAF3 and TRAF5 were overexpressed in the colon, while only TRAF5 increased in the small intestine of the experimental rats [8]. It seems that overexpression of TRAF5 induces expressions of adhesion molecules or inflammatory factors that contribute to the pathogenesis of inflammatory bowel disease. On the other hand, increased expression of TRAF3 may promote an exacerbated activation and survival of $\mathrm{T}$ cells, which may explain the inflammation and tissue damage in inflammatory bowel disease. Thus, TRAF3 and TRAF5 may play different roles in the pathogenesis of inflammatory bowel disease.

To date, the roles of TRAF3 and TRAF5 play in the inflammatory pathway is still debated. No evidence indicates expressions of TRAF3 and TRAF5 in human samples. The aim of the present study is to explore the potential roles of TRAF3 and TRAF5 in patients with inflammatory bowel disease. For this purpose, protein and gene levels of TRAF3 and TRAF5 expressions were detected in blood and tissue samples.

\section{Materials and Methods}

\section{Patients and Samples}

This retrospective case-control study was approved by the Research Ethics Committee of Renji Hospital, Shanghai Jiao-Tong University, School of Medicine. Written informed consents were obtained from all patients and healthy controls. All specimens were handled anonymously according to legal and ethical standards.

All patients were included based on clinical, endoscopical, and pathological proven by at least three gastroenterologists and diagnosis was not changed after at least 3 months of follow-up. Pregnancy, previous history of colon resection for ulcerative colitis, disease arising at only the small intestine, poor bowel preparation as visible area of intestinal mucosa $<90 \%$, and use of steroids or immunosuppressants before sampling were used as exclusion criteria.

Blood samples were obtained from subjects refrained from food or beverages for at least 12 hours before collection. Four tissue specimens were collected from patients with inflammatory bowel disease and 2 tissue specimens were collected from healthy controls during the endoscopy, respectively. Two areas of inflamed colonic segments and two areas of normal mucosal segments which were at least $5 \mathrm{~cm}$ away from the lesions were obtained from patients with inflammatory bowel disease. Specimens met the criteria established by Elmunzer [9], and specimens were snap frozen in liquid nitrogen within 10 mins after biopsy.

\section{Enzyme-linked Immunosorbent Assay (ELISA)}

Blood samples were collected in commercially EDTA-treated tubes (Gongdong Medical Technology Co., Ltd). Plasma was obtained by centrifugation for 15 minutes at 2,000 $\times \mathrm{g}$ using a refrigerated centrifuge. Samples were stored at $-80^{\circ} \mathrm{C}$. Plasma levels of TRAF3 and TRAF5 were analyzed using commercially available ELISA kit (ADL, San Diego CA, USA) according to the manufacturer's protocol. Specimens and standards were dispensed into appropriated wells and $50 \mu$ of Enzyme Conjugated Reagent was dispensed into each well. Wells were incubated at $37^{\circ} \mathrm{C}$ for 60 minutes after gently mixing. The incubation mixture was removed and the microtiter wells were rinsed 5 times. $50 \mu \mathrm{l}$ of color A and color B Reagent were dispensed into each well. Stop Solution was added to each well. A microtiter plate reader was used to read the absorbance at $450 \mathrm{~nm}$.

\section{Quantitative Real-time PCR (qRT-PCR)}

Peripheral blood mononuclear cells (PBMCs) were isolated from whole blood for subsequent RNA analysis using Lymphoprep ${ }^{\mathrm{TM}}$ reagent (Axis-Shield PoC AS). Total RNA in PBMCs and colon tissues were isolated using TRIzol ${ }^{\circledR}$ Reagent (Ambion) according to the manufacturer's protocol. All primers were synthesized at Invitrogen after design with PRIMER 5.0 (ABI). The primer set for TRAF3 was 5'-CTCACAAGTGCAGCGTCCAG-3' (forward) and 5'-GCTCCACTCCTTCAGCAGGTT-3' (reverse), with a product of 188 bps. The primer set for TRAF5 was 5'- CTGTGCTGTAACGGATAAACGG-3' (forward) and 5' - TAGCTGCTGGATTTTACTTTCTTTC-3' (re- 
verse), with a product of $160 \mathrm{bps}$. GAPDH was used as the internal control and the primer set was 5'-GTGAAGGTCGGAGTCAACGG-3' (forward) and 5'-CCTGGAAGATGGTGATGGGAT-3' (reverse), with a product of $226 \mathrm{bps}$. For gene-specific RT-PCR, first strand synthesis was performed with PrimeScript ${ }^{\mathrm{TM}} \mathrm{RT}$ reagent Kit (TaKaRa) following the manufacturer's specifications. Reverse transcriptions were performed at $37^{\circ} \mathrm{C}$ for 15 minutes and $85^{\circ} \mathrm{C}$ for 5 seconds. Quantitative real-time PCRs were done on a StepOne Plus device (Applied Biosystems) with SYBR Premix Ex Taq ${ }^{\mathrm{TM}}$ kit (TaKaRa). PCR amplification was carried out at $95^{\circ} \mathrm{C}$ for 10 seconds followed by $40 \mathrm{cy}-$ cles of $95^{\circ} \mathrm{C}, 5$ seconds and $60^{\circ} \mathrm{C}$ for 20 seconds. Data were analyzed by $2-\Delta \Delta \mathrm{Ct}$ method [10].

\section{Western Blot Analysis}

For Western blot analysis, proteins were isolated with Mem-PER ${ }^{\circledR}$ Membrane protein extraction reagent kit (Pierce) from frozen colonic tissues. The protein content of the samples was determined using Pierce ${ }^{\circledR}$ BCA Protein Assay Kit (Pierce). Equal amounts of $15 \mathrm{ug}$ protein were separated electrophoretically on $10 \%$ SDS-polyacrylamide gels and transferred onto polyvinylidene difluoride membranes (Roche). Thereafter, the membranes were sequentially probed with primary and secondary antibodies. Briefly, the membranes were probed with rabbit polyclonal anti-human TRAF3 (Santa Cruz, 1:1000), rabbit polyclonal anti-human TRAF5 (Santa Cruz, 1:1000) and mouse monoclonal anti-human $\beta$-actin (Santa Cruz, 1:2500). The expression levels of TRAF3 and TRAF5 were determined by incubating the membranes with goat anti-rabbit IgG-HPR (Santa Cruz,
1:10000) according to the manufacturers' instruction. After secondary antibody incubation and wash, the protein bands were visualized using SuperSignal ${ }^{\circledR}$ West Pico Chemiluminescent Substrate (Pierce).

\section{Statistical Analysis}

For statistical analysis, GraphPad Prism 5.0 for Windows (GraphPad Software, San Diego CA, USA) was used. Data is expressed as mean and $95 \%$ confidence intervals (CIs). P values were considered statistically significant when less than 0.05 . Discrete variables were presented as frequency and a two-sided Fisher's exact test or $\mathrm{X}^{2}$ test was performed to analyze associations between TRAFs' expressions and clinical parameters. Multiple group comparisons were done by one-way ANOVA and posthoc analysis when significantly different.

\section{Results}

\section{Characteristics of Included Subjects}

A total of 40 patients with Crohn's disease, 42 patients with ulcerative colitis, and 40 healthy controls were enrolled in this study from February 2007 to February 2010. The characteristics of these enrolled subjects are summarized in Table 1. Patients with Crohn's disease or ulcerative colitis exhibited significantly lower body mass index than healthy controls $(P<0.0001)$. The number of smoker is significantly higher in patients with ulcerative colitis than in Crohn's disease $(P=0.0023)$. However, no significant differences were found between patients and healthy controls in either age or gender.

Table I. Characteristics of Included Subjects.

\begin{tabular}{llll}
\hline & $\mathrm{CD}(n=40)$ & $\mathrm{UC}(n=42)$ & $\mathrm{HC}(40)$ \\
\hline Gender (Female/Male) & $21 / 19$ & $20 / 22$ & $20 / 20$ \\
Age (years) & $33.58(28.87-38.28)$ & $41.64(37.18-46.10)$ & $35.48(30.06-40.89)$ \\
BMI (kg/m $\left.{ }^{2}\right)$ & $19.29(18.81-19.76)^{* * *}$ & $20.00(19.41-20.59)^{* * *}$ & $22.84(22.10-23.58)$ \\
Smoking (Yes/No) & $8 / 32$ & $22 / 20^{* *}$ & No data
\end{tabular}

Extent

Ileitis 3

Ileocolitis 15

Colitis 22

Proctosigmoiditis 13

Left sided colitis $\quad 20$

Pancolitis 9

Endoscopic Score† 3.200(1.650-6.000) 2.000(1.000-3.000)

$\mathrm{CD}$, Crohn's disease; UC, ulcerative colitis; HC, healthy controls; BMI, body mass index; ${ }^{* * *}, P<0.0001$, significance is the difference from healthy controls; ${ }^{* *}$, $P<0.01$, significance is the difference from patients with Crohn's disease; $\dagger$, values are medians and $25 \%-75 \%$ percentile, simplified endoscopic score in Crohn's disease (SES-CD) is used to validate endoscopic severity in Crohn's disease, and Baron score is used to validate endoscopic severity in ulcerative colitis respectively. 
Soluble TRAF3 and TRAF5 increase in plasma of patients with Crohn's disease and ulcerative colitis

We examined the expressions of soluble TRAF3 and TRAF5 in plasma of patients with Crohn's disease and ulcerative colitis. The protein levels of TRAF3 and TRAF5 were significantly higher both in patients with Crohn's disease and ulcerative colitis than in healthy controls (Fig 1A and 1B). However, only soluble TRAF5 showed a weak correlation with endoscopic disease activity index (Baron score) in patients with ulcerative colitis (spearman's $r=0.358, P=0.022$ ). And no significant differences were found in protein expressions of soluble TRAF3 and TRAF5 between patients with Crohn's disease and patients with ulcerative colitis.

\section{TRAF3 and TRAF5 gene expression increase in peripheral blood mononuclear cells}

Peripheral blood mononuclear cells were isolated to determine the gene expressions of TRAF3 and TRAF5. Copy number of mRNA for each gene was accessed using quantitative real-time PCR. The gene expressions of TRAF3 and TRAF5 were significantly higher both in patients with Crohn's disease and ulcerative colitis than in healthy controls (Fig 1C and
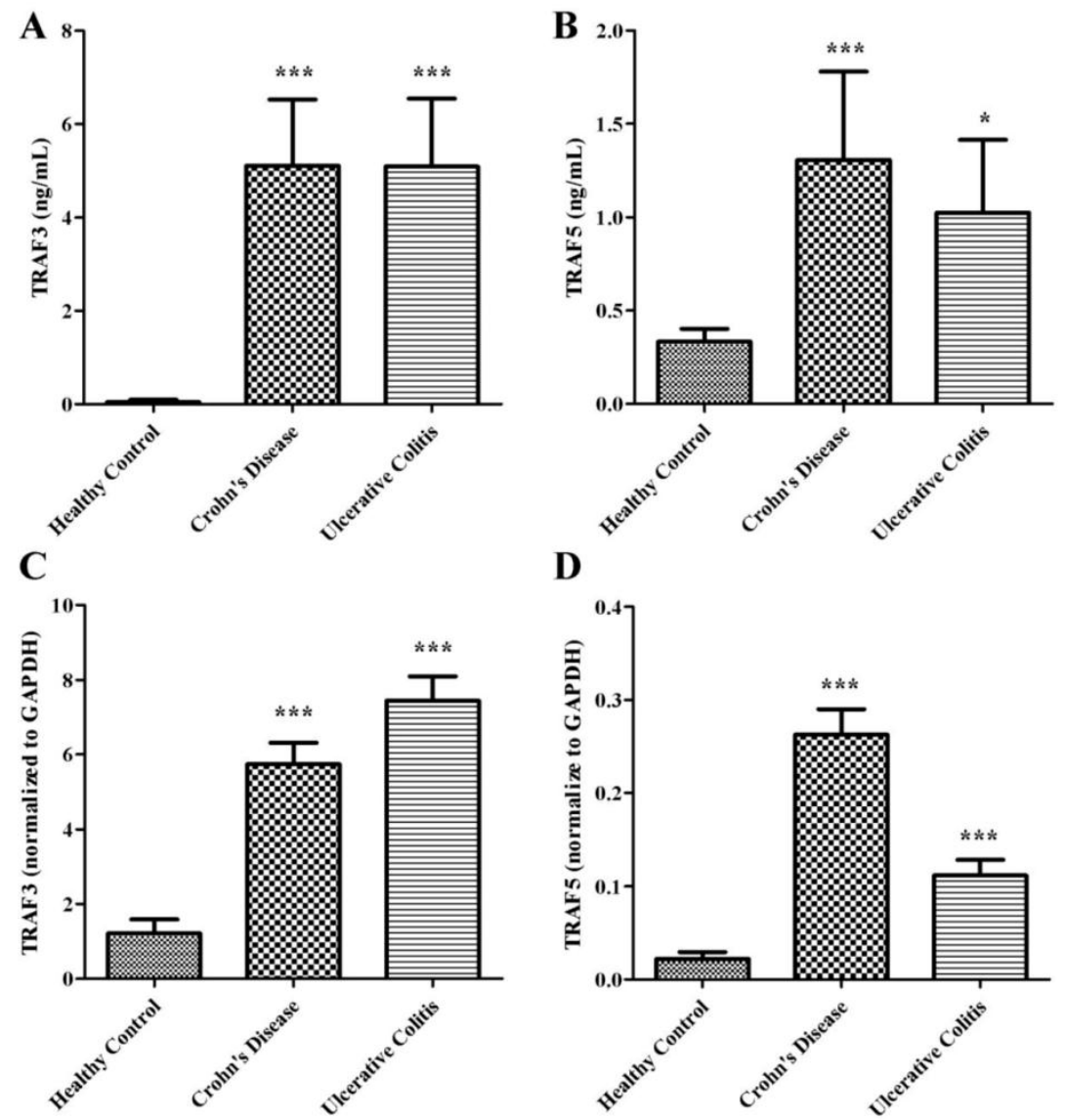

1D) (all $P<0.0001)$, which were similar to protein expressions in plasma of patients.

\section{Up-regulation and pre-activation of TRAF3 and TRAF 5 proteins in colonic tissues of pa- tients with Crohn's disease and ulcerative co- litis}

It is clear that inflammatory bowel disease exhibits segmental changes in the inflammatory colon. When appropriate methods used to analyze intestinal mucosa, even intestinal segments in remission can appear microscopic colitis [11]. Therefore, we compared the protein expressions of TRAF3 and TRAF5 both in inflamed and non-inflamed intestinal mucosa in patients with inflammatory bowel disease. 40 healthy controls, 32 patients with Crohn's disease and 30 patients with ulcerative colitis were included. Three patients with ileitis in Crohn's disease group and 9 patients with pancolitis were excluded. Five patients with Crohn's disease and 3 patients with ulcerative colitis refused to provide colonic tissue samples. Tissue samples from inflamed and non-inflamed intestinal mucosa were isolated and TRAF3 and TRAF5 proteins were measured via western blot analysis (Fig 2). It was found that TRAF3 and TRAF5 expressions were significantly higher in inflamed intestinal mucosa of patients than in non-inflamed intestinal mucosa and normal mucosa of healthy controls (Fig 3A and 3B). Furthermore, TRAF3 and TRAF5 expressions were also significantly higher in non-inflamed intestinal mucosa of patients with Crohn's disease and ulcerative colitis than in normal mucosa of healthy controls, which may indicate potential pre-activation of TRAF3 and TRAF5 proteins in colonic tissues of patients with Crohn's disease and ulcerative colitis.

Figure I. Soluble TRAF3 (A) and TRAF5 (B) protein levels in plasma, TRAF3 (C) and TRAF5 (D) gene expression in peripheral blood mononuclear cells of patients with Crohn's disease and ulcerative colitis. $* P<0.01 * * * P<0.000$ I 


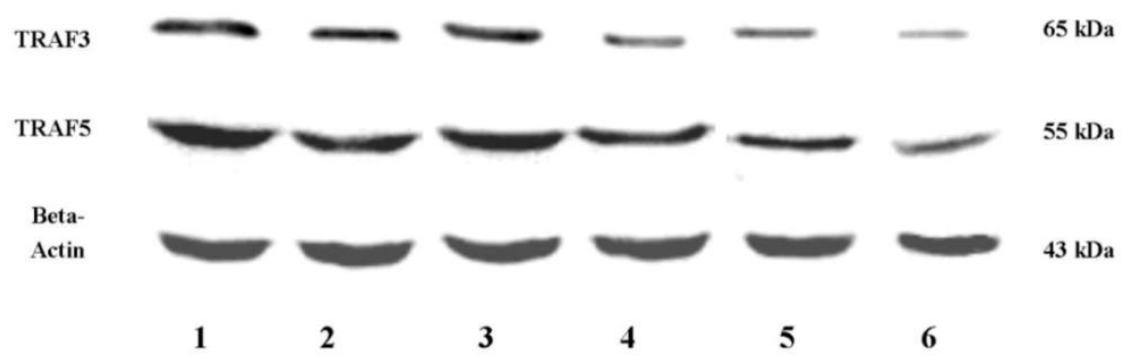

Figure 2. Western blot analyses of TRAF3 and TRAF5 protein expressions in colon. Abbreviations: CD, Crohn's disease; UC, ulcerative colitis; HC, healthy controls; I, 3: inflamed colonic mucosa; 2, 4: non-inflamed intestinal mucosa under endoscopy; 5, 6: normal colonic mucosa in healthy controls under endoscopy
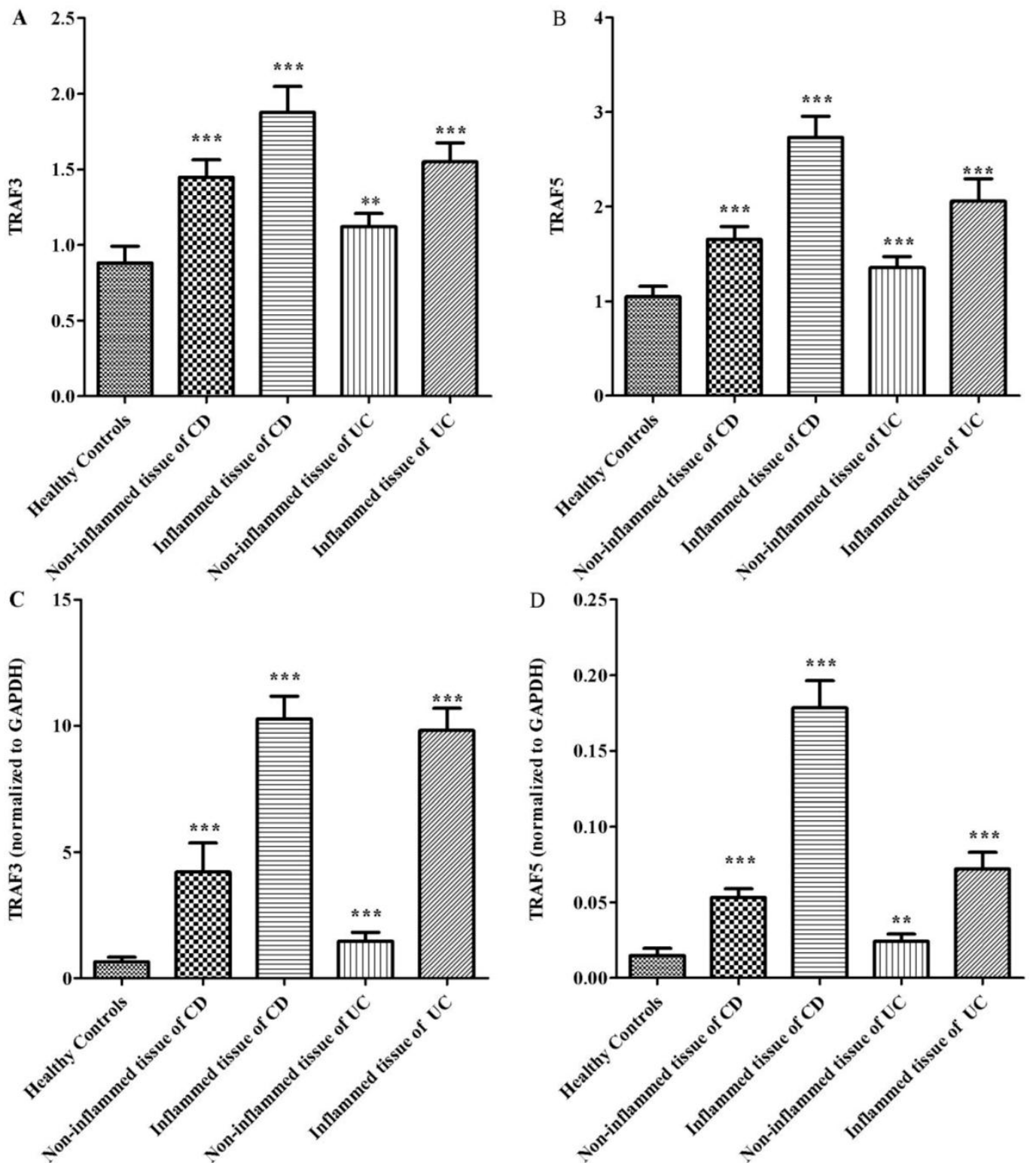

Figure 3. Differences in protein and gene expressions of TRAF3-TRAF5 in the inflamed and non-inflamed colonic mucosa in patients with Crohn's disease, ulcerative colitis and in normal control tissues. A, TRAF3 protein expression; B, TRAF5 protein expression; C, TRAF3 gene expression, D, TRAF5 gene expression. $* * P<0.001 * * * P<0.0001$ 


\section{Up-regulation and pre-activation of TRAF3 and TRAF5 gene expression in colonic tissues of patients with Crohn's disease and ulcerative colitis}

The gene expressions of TRAF3 and TRAF5 were determined using quantitative real-time PCR. Similar to protein expressions, TRAF3 and TRAF5 gene expressions were significantly higher in inflamed intestinal mucosa of patients than in non-inflamed mucosa and normal mucosa of healthy controls (Fig 3C and 3D). TRAF3 and TRAF5 gene expressions were also significantly higher in non-inflamed intestinal mucosa of patients with Crohn's disease and ulcerative colitis than in normal mucosa of healthy controls.

\section{Discussion}

Inflammatory bowel diseases present as life-long recurring inflammatory disorders of the gastrointestinal tract mediated by mucosal immune abnormalities [12]. The natural course of disease can range from prolonged periods of remission to aggressive, incapacitating disease [13]. In another sense, Crohn's disease and ulcerative colitis can be characterized by segmental inflammation of the gut [14]. Unaffected intestine may also show inflammatory changes with progression of disease. Thus, our present study attempts to give several aspects for TRAF3 and TRAF5 pre-activation in non-inflamed colonic segments and plasma. There are several reasons why TRAF3 and TRAF5 pre-activation show critical roles in inflammatory bowel disease. First, TRAF3 and TRAF5 expression can identify individuals at risk for the disease and can be disease specific. Combination of TRAF3 and TRAF5 pre-activation has a prognostic value towards relapse or recurrence of inflammatory bowel disease. Second, analysis of TRAF3 and TRAF5 expression can not only facilitate clinician's choice of the optimal initial therapy but may also be useful for adjusting treatment. For instance, patients in remission with combination therapy who have a low TRAF3 and TRAF5 expression in non-inflamed colonic segments and plasma may be able to de-escalate to a single-agent regimen. However, patients in remission with high TRAF3 and TRAF5 expression in non-inflamed colonic segments and plasma present probability of relapse or disease progression, which possibly increases the duration for maintaining remission. Therefore, pathogenesis predicting which group of patients is more susceptible to developing severe intestinal lesions is important for choosing suitable treatment strategies.

TRAFs originally emerged as signal transducing molecules for the TNF receptor superfamily members
[15]. Recently, it has been recognized that TRAFs is crucial for the activation of downstream immune response genes and proteins [16]. TRAF5 is structurally similar to TRAF3. However, TRAF3 is constitutively expressed in most cell types, whereas TRAF5 is mainly expressed in immune cells. Moreover, TRAF3 and TRAF5 are found to be involved in different signaling pathways, including NF-kB, MAPK, TLRs and IL-1R families. It is found that both TRAF3 and TRAF5 were overexpressed in colon of experimental rats, while only TRAF5 was overexpressed in the small intestine [8]. To our knowledge, we are the first to measure the levels of TRAF3 and TRAF5 in patients with inflammatory bowel disease. Our data indicate that TRAF3 and TRAF5 are overexpressed in plasma, PBMCs and colonic mucosa of patients with inflammatory bowel disease. Potential pre-activation of TRAF3 and TRAF5 is detected in non-inflamed colonic tissues of patients with Crohn's disease and ulcerative colitis. Initial expression experiments suggest that TRAF3 plays negative regulatory roles in CD40-stimulated classical NF-KB signaling and TNFR-induced MAPK activation $[17,18]$. However, based on more recent evidence it appears that TRAF3 is involved in both positive and negative regulatory functions of immune and inflammatory responses. Consistent with results from TRAF3-deficient mice, patients with reduced TRAF3 levels exhibit defects in type I IFN expression, which indicates that TRAF3 has an essential role in type I IFN induction and immunity [19]. A key insight into TRAF3 function was provided in that TRAF3 is constitutively bound to NF-KB inducing kinase (NIK), an essential activator of the alternative NF-кB pathway in unstimulated B cells for regulation of homeostasis [20]. Thus, overexpression of TRAF3 might be an outcome of feedback inhibition that restrains B-cell survival in inflammatory bowel disease instead of serving as a negative regulator of BAFF receptor (BAFF-R). Interestingly, B-cell activation by the cytoplasmic tail of latent membrane protein 1 (LMP1) is TRAF3 dependent on recruiting TRAF5 to the LMP1 signaling complex $[21,22]$. Although TRAF3 is suppressed in cellular survival pathways of $B$ cells, evidence suggests that $T$ cells deficient in TRAF3 might be dysfunctional [23]. In early stages of T-cell signaling, TRAF3 is needed by TCR and CD28 signaling complex for costimulation with anti-CD3 and anti-CD28 antibodies, which subsequently induces TCR/CD28-mediated early signaling events [24]. Therefore, as discussed above, it is now assumed that overexpressed TRAF3 may act as feedback activation in $\mathrm{T}$ cell and B cell signaling.

TRAF5 was been shown to interact with TRAF3 in recruitment to NF-KB, CD40 or LMP1 pathways via 
homotypic multimers or heterotypic multimers $[25,26]$, sparking our interest in the combined analysis of TRAF3 and TRAF5. TRAF5 is mainly expressed in immune cells and participates in the regulation of NF- $\mathrm{kB}$, JNK and interferon-regulated factor (IRF) signaling pathways $[27,28]$. Furthermore, TRAF5 can be considered a positive regulator of signaling downstream of costimulatory molecules as CD 27 and CD30 $[29,30]$. Meanwhile, abnormal NF-kB, JNK and IRF signaling pathways lead to deregulation of the inflammatory response and induction of immune defense, which play important roles in pathogenesis of inflammatory bowel disease [31,32]. Additionally, JNK activation, Akt phosphorylation, and the expression of IL-6, TNFa and IL-17 of splenic B cells are significantly decreased in LMP1+TRAF5\%- mice compared to $\mathrm{LMP1}^{+} \mathrm{TRAF}^{+/+}$mice [33]. LMP1-transgenic mice also exhibit B-cell hyperactivity. LMP1 excessively activates CD40 signaling as a functional mimic of CD40, which contributes to the pathogenesis of human autoimmune disease [34]. These results indicate that TRAF5 may be essential for LMP1-mediated signaling.

To conclude, TRAF3 and TRAF5 are overexpressed in inflammatory bowel disease. Although the endoscopic appearance can be normal, potential pre-activation of TRAF3 and TRAF5 is detected in non-inflamed colonic segments. Though there are still doubts regarding the function of TRAF3 and TRAF5, they are emerging as important regulators of immune cells in autoimmune diseases. Besides, TRAF3 and TRAF5 show potential roles in predicting aggressive disease, which can be used to evaluate response to treatment, choose therapeutic agents and treatment strategies. However, mechanisms responsible for segmental inflammation in inflammatory bowel disease remain to be fully understood. Thus, further mechanisms of TRAF3 and TRAF5 signaling in inflammatory bowel disease will be valuable for understanding TRAF3-TRAF5 interaction and segmental lesions in the pathogenesis of inflammatory bowel disease.

\section{Acknowledgements}

This work was supported by the National Natural Science Foundation of China (No. 81000161 and No.81170362), and Shanghai Jiao-Tong University School of Medicine Technology Fund (No. YZ1036). We would like to appreciate Dr. Lindholm Christopher from University of Chicago revised the language and discussion of this paper.

\section{Competing Interests}

The authors have declared that no competing interest exists.

\section{References}

1. Chung JY, Park YC, Ye H, et al. All TRAFs are not created equal: common and distinct molecular mechanisms of TRAF-mediated signal transduction. J Cell Sci. 2002;115: 679-688.

2. Yu X, Yi H, Guo C, et al. Pattern recognition scavenger receptor CD204 attenuates Toll-like receptor 4-induced NF-kappaB activation by directly inhibiting ubiquitination of tumor necrosis factor (TNF) receptor-associated factor 6. J Biol Chem. 2011; 286:18795-18806.

3. Wixted JH, Rothstein JL, Eisenlohr LC. Identification of functionally distinct TRAF proinflammatory and phosphatidylinositol 3-kinase/mitogen-activated protein kinase/extracellular signal-regulated kinase kinase (PI3K/MEK) transforming activities emanating from RET/PTC fusion oncoprotein. J Biol Chem. 2012; 287: 3691-3703.

4. Hildebrand JM, Yi Z, Buchta CM, et al. Roles of tumor necrosis factor receptor associated factor 3 (TRAF3) and TRAF5 in immune cell functions. Immunol Rev. 2011; 244:55-74.

5. Grech A, Quinn R, Srinivasan D, et al. Complete structural characterisation of the mammalian and Drosophila TRAF genes: implications for TRAF evolution and the role of RING finger splice variants. Mol Immunol. 2000;37:721-734.

6. Arcipowski KM, Stunz LL, Graham JP, et al. Molecular mechanisms of TNFR-associated factor 6 (TRAF6) utilization by the oncogenic viral mimic of CD40, latent membrane protein 1 (LMP1). J Biol Chem. 2011;286:9948-9955.

7. Hauer J, Püschner S, Ramakrishnan $\mathrm{P}$, et al. TNF receptor (TNFR)-associated factor (TRAF) 3 serves as an inhibitor of TRAF2/5-mediated activation of the noncanonical NF-kappaB pathway by TRAF-binding TNFRs. Proc Natl Acad Sci U S A. 2005;102:2874-2879.

8. Rojas-Cartagena C, Flores I, Appleyard CB. Role of tumor necrosis factor receptors in an animal model of acute colitis. Cytokine. 2005;32:85-93.

9. Elmunzer BJ, Higgins PD, Kwon YM, et al. Jumbo forceps are superior to standard large-capacity forceps in obtaining diagnostically adequate inflammatory bowel disease surveillance biopsy specimens. Gastrointest Endosc. 2008; 68:273-278.

10. Livak KJ, Schmittgen TD. Analysis of relative gene expression data using real-time quantitative PCR and the 2(-Delta Delta C(T)) Method. Methods. 2001;25:402-408.

11. Lasson A, Kilander A, Stotzer PO. Diagnostic yield of colonoscopy based on symptoms. Scand J Gastroenterol. 2008; 43:356-362.

12. Kugathasan S, Saubermann LJ, Smith L, et al. Mucosal T-cell immunoregulation varies in early and late inflammatory bowel disease. Gut. 2007; 56:1696-1705.

13. Yarur AJ, Strobel SG, Deshpande AR, et al. Predictors of aggressive inflammatory bowel disease. Gastroenterol Hepatol (N Y). 2011;7:652-659.

14. Yamazaki K, Onouchi Y, Takazoe M, et al. Association analysis of genetic variants in IL23R, ATG16L1 and 5p13.1 loci with Crohn's disease in Japanese patients. J Hum Genet. 2007;52:575-583.

15. Arch RH, Gedrich RW, Thompson CB. Tumor necrosis factor receptor-associated factors (TRAFs) a family of adapter proteins that regulates life and death. Genes Dev 1998;12:2821-2830.

16. Häcker $\mathrm{H}$, Tseng PH, Karin M. Expanding TRAF function: TRAF3 as a tri-faced immune regulator. Nat Rev Immunol. 2011;11:457-468.

17. Cheng G, Cleary AM, Ye ZS, et al. Involvement of CRAF1, a relative of TRAF, in CD40 signaling. Science. 1995;267:1494-1498.

18. Tseng PH, Matsuzawa A, Zhang W, et al. Different modes of ubiquitination of the adaptor TRAF3 selectively activate the expression of type I interferons and proinflammatory cytokines. Nat Immunol. 2010;11:70-75.

19. Pérez de Diego R, Sancho-Shimizu V, Lorenzo L, et al. Human TRAF3 adaptor molecule deficiency leads to impaired Toll-like receptor 3 response and susceptibility to herpes simplex encephalitis. Immunity. 2010;33:400-411.

20. Malinin NL, Boldin MP, Kovalenko AV, et al. MAP3K-related kinase involved in NF-kappaB induction by TNF, CD95 and IL-1. Nature. 1997;385:540-544.

21. Xie P, Hostager BS, Bishop GA. Requirement for TRAF3 in signaling by LMP1 but not CD40 in B lymphocytes. J Exp Med. 2004;199:661-671.

22. Arcipowski KM, Stunz LL, Graham JP, Kraus ZJ, Vanden Bush TJ, Bishop GA. Molecular mechanisms of TNFR-associated factor 6 (TRAF6) utilization by the oncogenic viral mimic of $\mathrm{CD} 40$, latent membrane protein 1 (LMP1). J Biol Chem. 2011;286:9948-9955. 
23. $\mathrm{Xu} \mathrm{Y,} \mathrm{Cheng} \mathrm{G,} \mathrm{Baltimore} \mathrm{D.} \mathrm{Targeted} \mathrm{disruption} \mathrm{of} \mathrm{TRAF3} \mathrm{leads} \mathrm{to}$ postnatal lethality and defective T-dependent immune responses. Immunity. 1996;5:407-415.

24. Xie P, Kraus ZJ, Stunz LL, et al. TNF receptor-associated factor 3 is required for T cell-mediated immunity and TCR/CD28 signaling. J Immunol. 2011;186:143-155.

25. Pullen SS, Miller HG, Everdeen DS, et al. CD40-tumor necrosis factor receptor-associated factor (TRAF) interactions: regulation of CD40 signaling through multiple TRAF binding sites and TRAF hetero-oligomerization. Biochemistry. 1998;37:11836-11845.

26. He L, Grammer AC, Wu X, et al. TRAF3 forms heterotrimers with TRAF2 and modulates its ability to mediate NF-\{kappa\}B activation. J Biol Chem. 2004;279:55855-55865.

27. Kraus ZJ, Nakano H, Bishop GA. TRAF5 is a critical mediator of in vitro signals and in vivo functions of LMP1, the viral oncogenic mimic of CD40. Proc Natl Acad Sci USA 2009;106:17140-17145.

28. Missiou A, Rudolf P, Stachon P, et al. TRAF5 deficiency accelerates atherogenesis in mice by increasing inflammatory cell recruitment and foam cell formation. Circ Res. 2010;107:757-766.

29. Nakano H, Sakon S, Koseki H, et al. Targeted disruption of Traf5 gene causes defects in CD40- and CD27-mediated lymphocyte activation. Proc Natl Acad Sci U S A. 1999;96:9803-9808.

30. Aizawa S, Nakano $H$, Ishida $T$, et al. Tumor necrosis factor receptor-associated factor (TRAF) 5 and TRAF2 are involved in CD30-mediated NFkappaB activation. J Biol Chem. 1997;272:2042-2045.

31. Wei J, Feng J. Signaling pathways associated with inflammatory bowel disease. Recent Pat Inflamm Allergy Drug Discov. 2010;4:105-117.

32. Haller D. Intestinal epithelial cell signalling and host-derived negative regulators under chronic inflammation: to be or not to be activated determines the balance towards commensal bacteria. Neurogastroenterol Motil. 2006;18:184-199.

33. Stunz LL, Busch LK, Munroe ME, et al. Expression of the cytoplasmic tail of LMP1 in mice induces hyperactivation of B lymphocytes and disordered lymphoid architecture. Immunity. 2004;21:255-266.

34. Graham JP, Arcipowski KM, Bishop GA. Differential B-lymphocyte regulation by $\mathrm{CD} 40$ and its viral mimic, latent membrane protein 1 . Immunol Rev. 2010;237:226-248. 